\title{
Approximate Analysis of Arbitrary QNMs with Space and Service Priorities
}

\author{
Irfan-Ullah Awan and Demetres D. Kouvatsos \\ Computer Systems Modelling Research Group, \\ University of Bradford \\ Bradford BD7 1DP, U.K. \\ email \{I.Awan, D.D.Kouvatsos\}@scm.brad.ac.uk
}

\begin{abstract}
Closed-form solutions are characterised, based on the principle of maximum entropy (ME), for a single server censored $\mathrm{G} / \mathrm{G} / 1 / \mathrm{N}_{1}, \ldots, \mathrm{N}_{R}$ queue with $R(R>1)$ priority classes under Head-of-Line (HOL) service discipline and Partial Buffer Sharing (PBS) scheme. The forms of the joint, aggregate and marginal state probabilities, as well as basic performance measures such as mean queue length and cell loss probability are analytically established at equilibrium via appropriate mean value constraints and the generating function approach. Consequently, efficient recursive expressions of low computational cost are determined. Furthermore, the G/G/1/ $\mathrm{N}_{1}, \ldots, \mathrm{N}_{R}$ queue is utilised, in conjunction with appropriate flow approximation formulae, as a cost-effective building block towards a queue-by-queue decomposition algorithm of arbitrary open queueing network models (QNMs) under repetitive service blocking with random destination (RS-RD). Typical numerical results are included to illustrate the credibility of the approximate algorithm against simulation in the context of Generalised Exponential (GE)-type buffered interconnection networks with bursty traffic.
\end{abstract}

\section{Keywords}

Queueing Network Model (QNM), Repetitive Service Blocking with Random Destination (RS-RD), Maximum Entropy (ME) Principle, Head-of-Line (HOL) Service Discipline, Partial Buffer Sharing (PBS) Scheme, Generalised Exponential (GE) Distribution, Asynchronous Transfer Mode (ATM) Networks.

*Supported in part by the Engineering and Physical Sciences Research Council (EPSRC), UK, under grants GR/H/18609 and GR/K/67809 and in part by British Council under ODA scheme.

The original version of this chapter was revised: The copyright line was incorrect. This has been corrected. The Erratum to this chapter is available at DOI: 10.1007/978-0-387-35353-1_28 


\section{INTRODUCTION}

Finite buffer queues and network models with service and space priorities are of great importance towards effective congestion control and quality of service (QoS) protection in Asynchronous Transfer Mode (ATM) networks.

An ATM cell can be either of high or low priority depending on whether the cell loss priority (CLP) bit in the cell's header has been set or not. A cell of high priority has by default its CLP bit set to zero. The CLP bit of low priority cells is set to one. It is the job of the priority mechanism to monitor the CLP bit of arriving cells and give preferential treatment to high priority cells. Priority mechanisms include time priorities and space priorities.

Time priority mechanisms such as Head-of-Line (HOL) e.g., (Hong and Suda 1991) and Preemptive Resume (PR) e.g., (Kouvatsos and Tabet-Aouel 1990), take into account that some services may tolerate longer delays than others (e.g., data versus voice) and deal with the order with which cells are transmitted. Although time priorities are not explicitly identified in ITU Recommendation I.361 c.f., (ITU Draft Recommendations 1990), they can be implicitly represented by using combinations of virtual path and channel identifiers (VPI/VCI).

Space prioritiy mechanisms control the allocation of buffer space to arriving cells at an input or output port queue of an ATM switch. Implicitly, they provide several grades of service through the selective discarding of low priority cells. This type of priority mechanism exploits the fact that certain cells generated by traffic sources are less important than others and may, therefore, be discarded without significantly affecting the QoS constraints. Space priority mechanisms aim to decrease the cell loss probability and delays for high priority cells in comparison with low priority cells. Two main mechanisms for space priorities are push-out and partial buffer sharing (PBS).

Under a push-out mechanism e.g., (Sumita and Ozawa 1988), (Hebuterne and Gravey 1989), (Fourneau, Pekergin, and Taleb 1994), (Lin and Silvester 1993), low priority cells finding on arrival the buffer of the queue full will be lost immediately. High priority cells arriving at a full buffer queue of capacity $N$ will either replace (push-out) low priority cells from the queue (which are lost), if any, or are lost if only high priority cells are present (c.f., Fig. 1). Note that a combination of time and push-out priorities have been studied by (Gravey and Hebuterne 1991) and (Nillson, Lai and Perros 1990).

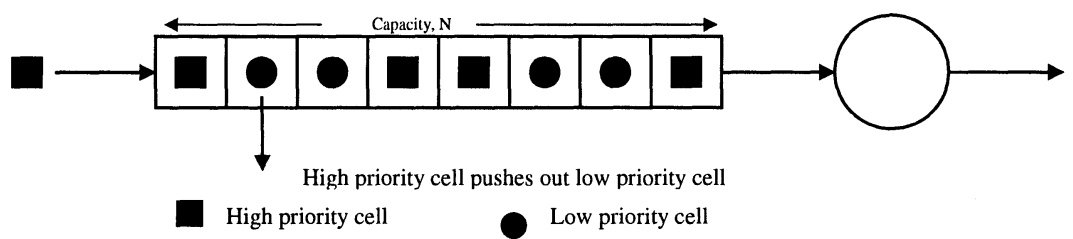

Fig. 1: The push-out space priority mechanism 
PBS works by setting a sequence of thresholds $N_{i}, i=1,2, \ldots, R$, corresponding to $R$ priority classes (indexed from 1 to $R$ in decreasing order of priority) of a single queue with finite capacity $N_{1}$. Highest priority cells of class $i=1$ can join the queue simply if there is space. However, lower priority cells of class $j, j=2, \ldots, R$, can join the queue only if the total number of cells in the queue is less than the threshold value $N_{j}$. Once the number of cells waiting for service reaches $N_{j}$, then all lower priority cells of class $k, k=j+1, \ldots, R$, will be lost on arrival but higher priority cells of class $i, i=1, \ldots, j-1$, will continue to join the queue until it reaches threshold value, $N_{i}, i=1, \ldots, j-1$ (c.f., for $R=2$ classes, see Fig. 2). Unlike push-out mechanism, once a cell of any class has been in place, it cannot be lost. Different cell loss and QoS requirements under various load conditions can be met by adjusting the threshold value. (Li 1989) and (Yin, Li and Stern 1990) applied PBS and selective packet discarding for the overload control of packet voice systems modelled by $\mathrm{M} / \mathrm{PH} / 1 / \mathrm{N}$ and $\mathrm{PH} / \mathrm{M} / 1 / \mathrm{N}$ queues. An $\mathrm{M} / \mathrm{M} / \mathrm{G} / 1 / \mathrm{N}$ queueing system employing PBS scheme was studied by (Kroner 1989).

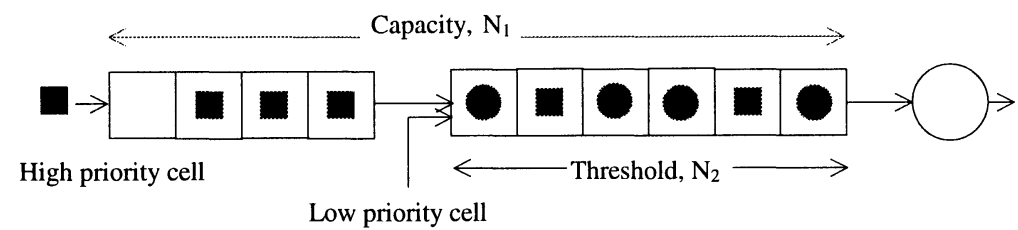

Fig. 2: The partial buffer sharing space priority mechanism

Some of the limitations associated with the analysis of priority mechanisms are due to exponential or deterministic arrivals which limit their usefulness whilst numerical and simulation methods for more general models may lead to time consuming (or even intractable) solutions as the system's state-space increases. These solutions also fail to reveal explicit functional relationships amongst performance metrics. Furthermore, most of the analytic space (and time) priority models proposed in the literature focus on a single server queue.

In this paper, the principle of maximum entropy (ME) (Jaynes 1957a) and the generating function approach (Williams and Bhandiwad 1976) are applied to characterise recursive expressions for state probabilities and basic performance measures for a general single server queue at equilibrium with finite capacity, $R(R>1)$ priority classes under Head-of-Line (HOL) service discipline and PBS scheme. Consequently, this queue is used, in conjunction with appropriate flow approximation formulae, as a cost-effective building block towards a queue-by-queue decomposition algorithm of arbitrary open queueing network models (QNMs) under repetitive service blocking with random 
destination (RS-RD). Validation tests involving ME and simulation results are carried out in the context of simple space division ATM switch architectures represented by arbitrary Generalised Exponential (GE) - type multistage interconnection networks of switching components with single input/output port and output queueing. Note that under RS-RD blocking, when a cell upon service completion at queue $i$ attempts to join a destination queue $j$ whose capacity is full, it is rejected by queue $j$ and immediately receives another service at queue $i$. Each time a cell completes service at queue $i$, a downstream queue is selected independently of the previously chosen destination queue $j$.

The principle of $\mathrm{ME}$ provides a self-consistent method of inference for characterising an unknown but true probability distribution, subject to known (or known to exist) mean value constraints. In an information theoretic context (Jaynes 1957a), the ME solution corresponds to the maximum disorder of system states and thus is considered to be the least biased distribution estimate of all solutions that satisfy the system's constraints. In sampling terms, (Jaynes 1957b) has shown that, given the imposed constraints, the ME solution can be experimentally realised in overwhelmingly more ways than any other distribution. Major discrepancies between the ME distribution and the experimentally observed distribution indicate that important physical constraints have been overlooked. Conversely, experimental agreement with the ME solution represents evidence that the constraints of the system have been properly identified. Note that the methodology of ME has been applied under general conditions to analyse arbitrary QNMs with non-priority classes and finite capacity, e.g., (Kouvatsos and Denazis 1993). Details on entropy maximisation and systems modelling can be seen in (Kouvatsos 1994).

For illustration purposes, it is assumed that the traffic entering and flowing in the network is bursty and is represented by a Compound Poisson Process (CPP) with geometrically distributed bulk sizes (Kouvatsos 1994). This particular process implies a GE interarrival-time distribution of the form

$$
\begin{aligned}
F(t) & =P(X \leq t) \\
& =1-\tau e^{-\tau v t}, t \geq 0
\end{aligned}
$$

where $\tau=2 /\left(C^{2}+1\right), X$ is the inter-event time random variable and $\left\{1 / v, C^{2}\right\}$ are the mean and squred coefficient of variation (SCV) of the inter-event time distribution, respectively (c.f., Fig. 3). The choice of the GE distribution is

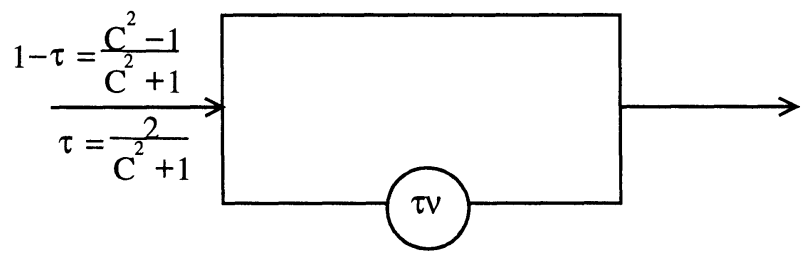

Fig. 3: The GE distribution with parameters $\tau$ and $\tau \nu(0 \leq \tau \leq 1)$ 
motivated by the fact that measurements of actual traffic or service times are generally limited and so only few parameters can be computed reliably. Typically, only the mean and variance can be relied upon, and thus, a choice of a distribution which imply least bias (i.e., introduction of arbitrary and, therefore, false assumptions) is that of GE-type distribution. In the context of ATM environment, this model is particularly applicable in cases of traffic with low level of correlation or where smoothing schemes are introduced at the adaptation level (e.g., for a stored video source) with the objective of minimising or even eliminating the problem of traffic correlation (Ball, Hutchinson and Kouvatsos 1996). Moreover, under renewality assumptions, the GE distribution is most appropriate to model simultaneous cell arrivals at output port queues generated by different bursty sources (e.g., voice or high resolution video) with known first two moments. In this context, the burstiness of the arrival process is characterised by the squared coefficient of variation (SCV) of the interarrival-time or equivalently, the size of the incoming bulk (c.f., (1)).

The form of the ME queue length distribution, subject to appropriate mean value constraints, for a single server censored queue at equilibrium with $R(R>1)$ HOL priority classes and PBS scheme is characterised in Section 2 together with recursive relationships describing performance metrics, based on the generating function approach (Williams and Bhandiwad 1976). This ME solution is used in Section 3 as a building block, in conjunction with flow approximation formulae, towards the creation of a queue-by-queue decomposition algorithm of arbitrary open QNMs under RS-RD blocking. A case study, based on the analysis of a single server GE-type queue with $R(R>1)$ HOL priority classes and PBS scheme, is carried out in Section 4. Validation tests involving GE-type ME and simulation results are given in Section 5. Conclusions follow in Section 6.

\section{ME ANALYSIS OF $\mathrm{G} / \mathrm{G} / 1 / \mathrm{N}_{1}, \ldots, \mathrm{N}_{R}$ QUEUE}

Consider an arbitrary single server queue at equilibrium with $(R \geq 2)$ HOL priority classes and PBS scheme denoted by $\mathrm{G} / \mathrm{G} / 1 / \mathrm{N}_{1}, \ldots, \mathrm{N}_{R}$ such that (i) the interarrival times and service times per class are generally $(G)$ distributed (ii) the total buffer capacity is $N_{1}$ and (iii) the PBS scheme is specified by the sequence of thresholds $\left\{N_{i}, i=2,3, \ldots, R\right\}$. Moreover, for each class $i, i=$ $1,2, \ldots, R$, the arrival process is assumed to be censored (i.e., a cell will be lost if on arrival it finds a full buffer) with mean rate $\lambda_{i}$ and interarrival time squared coefficient of variation (SCV), $C_{a i}^{2}$. Cells are transmitted by a single server with mean rate $\mu_{i}$ and SCV,$C_{s i}^{2}$.

Suppose that the priority classes are indexed from 1 to $R$ in decreasing order of priority. Let at any given time the state of the system be described by a vector $S \equiv\left(n_{1}, n_{2}, \ldots, n_{R}, \omega\right)$, where $n_{i}, i=1, \ldots, R$, is the number of class $i$ cells in the queue (waiting for or receiving service) and $\omega$ is the 
variable indicating the class of the current cell in service (n.b., for an idle queue $\omega=0$ ). Let $Q$ be the set of all feasible states $\{S\}$ and $P(S)$ be at any given time the equilibrium probability that the $\mathrm{G} / \mathrm{G} / 1 / \mathrm{N}_{1}, \ldots, \mathrm{N}_{R}$ priority queue is in state $S$ and $\pi_{i}$ be the cell loss probability that an arriving cell of class $i, i=1,2, \ldots, R$, will find the queue occupied up to at least its corresponding threshold $N_{i}, i=1,2, \ldots, R$.

\subsection{Prior Information}

For each state $S, S \in Q$, and class $i, i=1,2, \ldots, R$, the following auxiliary functions are defined:

$$
\begin{aligned}
n_{i}(S) & =\text { the number of class } i \text { cells present in state } S, \\
s_{i}(S) & = \begin{cases}1, & \text { if the cell in service is of class } i, \\
0, & \text { otherwise, }\end{cases} \\
h_{i}(S) & = \begin{cases}1, & \text { if } n_{i}>0, \\
0, & \text { otherwise, }\end{cases} \\
f_{i}(S) & = \begin{cases}1, & \text { if } \sum_{j=1}^{R} n_{j}(S)=N_{i}, \text { and } s_{i}(S)=1, \\
0, & \text { otherwise }\end{cases}
\end{aligned}
$$

Suppose, now, all that is known about the state probabilities $\{P(S)\}$ is that the following mean value constraints exist:

(i) Normalisation,

$$
\sum_{S \in Q} P(S)=1
$$

(ii) Server utilisation, $U_{i}$,

$$
\sum_{S \in Q} s_{i}(S) P(S)=U_{i}, 0<U_{i}<1, i=1,2, \ldots, R
$$

(iii) Busy state probability per class, $\theta_{i}, 0<\theta_{i}<1$,

$$
\sum_{S \in Q} h_{i}(S) P(S)=\theta_{i}, i=1,2, \ldots, R
$$

(iv) Mean queue length, $\left\langle n_{i}\right\rangle$,

$$
\sum_{S \in Q} n_{i}(S) P(S)=\left\langle n_{i}\right\rangle, U_{i}<\left\langle n_{i}\right\rangle<N_{i}, i=1,2, \ldots, R
$$

(v) Full buffer state probability, $\phi_{i}$,

$$
\sum_{S \in Q} f_{i}(S) P(S)=\phi_{i}, 0<\phi_{i}<1
$$


satisfying the flow balance equations, namely

$$
\lambda_{i}\left(1-\pi_{i}\right)=\mu_{i} U_{i}, i=1,2, \ldots, R .
$$

The choice of mean values (2) - (6) is based on the type of constraints used for the ME analysis of stable single class FCFS G/G/1/N queue (Kouvatsos 1986). Note that if additional constraints are used, it is no longer feasible to capture a closed-form ME solution. Such solution is an essential building block in the context of a cost-effective queue-by-queue decomposition algorithm for arbitrary QNMs. Conversely, the removal of one or more constraints from the set (2)- (6) will result into a ME solution with reduced accuracy c.f., (Jaynes 1957a), (Jaynes 1957b).

\subsection{Maximum Entropy Solution}

The form of the state probability distribution, $P(S), S \in Q$, can be characterised by maximising the entropy functional $H(P)=-\sum_{s} P(S) \log P(S)$, subject to constraints (2) - (6). By employing Lagrange's method of undetermined multipliers, the ME solution is expressed by

$$
P(S)=\frac{1}{Z} \prod_{i=1}^{R} g_{i}^{s_{i}(S)} \xi_{i}^{h_{i}(S)} x_{i}^{n_{i}(S)} y_{i}^{f_{i}(S)}, \forall S \in Q
$$

where $Z$ is the normalising constant and $\left\{g_{i}, \xi_{i}, x_{i}, y_{i}\right\}$ are the Lagrangian

coefficients corresponding to constraints (3) - (6), respectively. Furthermore, aggregating (8) over all feasible states $S \in Q$, the joint ME queue length distribution is given by:

$$
P(\underline{\mathrm{n}})=\frac{1}{Z} \prod_{i=1}^{R} x_{i}^{n_{i}(S)} \xi_{i}^{h_{i}(S)} \sum_{s=1 \wedge n_{s}>0}^{R} g_{s} y_{s}^{f_{s}(\underline{\mathrm{n}})}, 0 \leq n_{i} \leq N_{i}, \& \sum_{i=1}^{R} n_{i} \leq N_{1}
$$

where $\underline{\mathrm{n}}=\left(n_{1}, n_{2}, \ldots, n_{R}\right)$ and $P(\underline{0})=1 / Z$.

\subsection{Estimation of the Lagrangian Coefficients}

The Lagrangian coefficients $g_{i}, \xi_{i}$ and $x_{i}, i=1, \ldots, R$, can be approximately determined in terms of input parameters via closed form asymptotic expressions based on the ME solution of the corresponding G/G/1 HOL priority queue at equilibrium c.f.,(Kouvatsos and Tabet-Aouel 1990) and are given 
by:

$$
\begin{aligned}
x_{i} & =\frac{\left\langle n_{i}\right\rangle-\theta_{i}}{\left\langle n_{i}\right\rangle}, g_{i}=\frac{\rho_{i}}{(1-\rho)} \frac{\rho-\theta_{i}}{\theta_{i}-\rho_{i}} \prod_{i \neq l=1}^{R} \frac{\rho-\theta_{l}}{\rho-\rho_{l}}, \\
\xi_{i} & =\frac{\left(\theta_{i}-\rho_{i}\right)}{\left(\rho-\theta_{i}\right)} \frac{\left(1-x_{i}\right)}{x_{i}},
\end{aligned}
$$

where $\rho_{i}=\lambda_{i} / \mu_{i}, \rho=\sum_{i=1}^{R} \rho_{i}$ and constraints $\left\{\left\langle n_{i}\right\rangle, \theta_{i}\right\}$ are the associated performance metrics of the infinite capacity $\mathrm{G} / \mathrm{G} / 1$ HOL queue (Kouvatsos and Tabet-Aouel 1990).

Note that Lagrangian coefficients, $y_{i}, i=1,2, \ldots, R$, are determined via recursive exressions in Section 4.2 by making use of flow balance condition (7) and GE-type cell loss probabilities, $\pi_{i}, i=1,2, \ldots, R$.

\subsection{Recursive Relationships}

Let us define

$Q_{i}=\left\{S \in Q / s_{i}(S)=1, \forall i=1,2, \ldots, R\right\}$,

$S_{o}=\left\{S \in Q / n_{i}(S)=0, \forall i=1,2, \ldots, R\right\}$,

and $\forall i=1,2, \ldots, R, \& v=0,1, \ldots, N_{1}$

$$
\begin{aligned}
S_{i}^{N_{1}} & =\left\{S / S \in Q_{i}, 0 \leq n_{j} \leq N_{j}, j=1, \ldots, R \& \sum_{j=1}^{R} n_{j} \leq N_{1}\right\}, \\
A_{i}^{v} & =\left\{S / S \in Q_{i}, 0 \leq n_{j} \leq v ; n_{j} \leq N_{j}, j=1, \ldots, R \& \sum_{j=1}^{R} n_{j}=v\right\} .
\end{aligned}
$$

The ME solution for each state $S, S \in Q_{i}, i=1,2, \ldots, R$ is given by

$$
P(S)=\frac{1}{Z} g_{i} \xi_{i} x_{i}^{n_{i}} y_{i}^{f_{i}(S)}\left(\prod_{i \neq j=1}^{R} x_{j}^{n_{j}} \xi_{i}^{h_{j}(S)}\right), \forall S \in Q
$$


and since $U_{i}=P\left(Q_{i}\right)=\sum_{S \in Q} s_{i}(S) P(S)$ and $S_{i}^{N_{1}}=\bigcup_{v=1}^{N_{1}} A_{i}^{v}$, it follows that

$$
U_{i}=\frac{1}{Z} g_{i} \xi_{i} x_{i}\left(\sum_{v=0}^{N_{1}-1} y_{i}^{\delta(v)} \sum_{S \in A_{i}^{v}} x_{i}^{n_{i}} \prod_{i \neq j=1}^{R} x_{j}^{n_{j}} \xi_{i}^{h_{j}(S)}\right), i=1, \ldots, R,
$$

where $\delta(v)=1$, if $v \geq N_{i}-1$ or 0 , otherwise. A more suitable formula, for computing the complex sums of eq. (12), can be determined by using the generating function approach as follows:

Define

$$
\begin{aligned}
P_{j}(z) & =\sum_{n_{j}=0}^{N_{j}} x_{j}^{n_{j}} \xi_{j}^{h_{j}(S)} z^{n_{j}},|z| \leq 1, j=1, \ldots, R \\
G_{R}(z) & =\sum_{v=0}^{\infty} C_{R}(v) z^{v}=\prod_{j=1}^{R} P_{j}(z) \\
& =\prod_{j=1}^{R}\left(\frac{1-\left(1-\xi_{j}\right) x_{j} z-\xi_{j}\left(x_{j} z\right)^{N_{j}+1}}{1-x_{j} z}\right)
\end{aligned}
$$

It is observed that the coefficient of $z^{v}$ in $G_{R}(z)$ is given by

$$
\begin{aligned}
& C_{R}(v)=\sum_{S \in A_{j}^{v}} \prod_{j=1}^{R} x_{j}^{n_{j}} \xi_{j}^{h_{j}}(S)=G_{R}^{(v)}(0), \\
& \text { where } G_{R}^{(v)}(0)=\left.\frac{d^{v} G_{R}(z)}{d z^{v}}\right|_{z=0} .
\end{aligned}
$$

By enhancing this definition of z-transform, let us define

$$
\begin{aligned}
& P_{i}(z)=\sum_{n_{i}=0}^{N_{i}-1} x_{i}^{n_{i}} z^{n_{i}}=\frac{1-\left(x_{i} z\right)^{N_{i}}}{1-x_{i} z}, \\
& G^{(i)}(z)=\sum_{v=0}^{\infty} C^{(i)}(v) z^{v}=P_{i}(z) \prod_{i \neq j=1}^{R} P_{j}(z),
\end{aligned}
$$

where $P_{j}(z)$ is given from eq. (13). Thus, eq. (16) becomes

$$
G^{(i)}(z)=\frac{1-\left(x_{i} z\right)^{N_{i}}}{1-x_{i} z} \prod_{i \neq j=1}^{R} \frac{1-\left(1-\xi_{j}\right) x_{j} z-\xi_{j}\left(x_{j} z\right)^{N_{j}+1}}{1-x_{j} z}
$$


where $C^{(i)}(v)$ is given by

$$
C^{(i)}(v)=\sum_{S \in A_{i}^{v}} x_{i}^{n_{i}} \prod_{i \neq j=1}^{R} x_{j}^{n_{j}} \xi_{j}^{h_{j}}(S) .
$$

Moreover, the coefficients $C^{(i)}(v), v=0,1, \ldots, \& i=1,2, \ldots, R$, can be computed via the following recursive formula

$$
\begin{aligned}
C^{(i)}(v)= & \left(1-\xi_{i}\right) x_{i} C^{(i)}(v-1)+C(v)-x_{i}^{N_{i}} C\left(v-N_{i}\right) \\
& +x_{i}^{N_{i}+1} \xi_{i} C^{(i)}\left(v-N_{i}-1\right)
\end{aligned}
$$

with initial conditions $C^{(i)}(v)= \begin{cases}0, & v<0, \\ 1, & v=0,\end{cases}$

where $C(v)=C_{R}(v)$, and $C_{R}(v)$ can be calculated recursively by

$$
\begin{aligned}
C_{r}(v)= & x_{r} C_{r}(v-1)+C_{r-1}(v)-\left(1-\xi_{r}\right) x_{r} C_{r-1}(v-1) \\
& -\xi_{r} x_{r}^{N_{r}+1} C_{r-1}\left(v-N_{r}-1\right),
\end{aligned}
$$

with initial conditions $C_{r}(v)= \begin{cases}0, & v<0, \\ 1, & v=0, \\ \xi_{1} x_{1}^{v}, & v>0 .\end{cases}$

Substituting (18) into (12) yields

$$
U_{i}=\frac{1}{Z} g_{i} \xi_{i} x_{i}\left(\sum_{v=0}^{N_{1}-1} y_{i}^{\delta(v)} C^{(i)}(v)\right) .
$$

From eq. (21), the following expression for $Z$ is derived:

$$
Z=1+\sum_{i=1}^{R} g_{i} \xi_{i} x_{i}\left(\sum_{v=0}^{N_{1}-1} y_{i}^{\delta(v)} C^{(i)}(v)\right)
$$

\subsection{Aggregate State Probabilities}

Unconditioning expressions (21) over all classes, an expression for the aggregate utilisation, $U$, can be obtained with aggregate arrival rate $\lambda$. The aggregate probability distributions, $\left\{P(v), v=0,1, \ldots, N_{1}\right\}$, are given by

$$
\begin{aligned}
& P(0)=P\left(S_{o}\right)=\frac{1}{Z} \\
& P(v)=\frac{1}{Z} \sum_{i=1}^{R} g_{i} \xi_{i} x_{i} y_{i}^{\delta(v)} C^{(i)}(v-1), \forall v=1,2, \ldots, N_{1} .
\end{aligned}
$$




\subsection{Marginal State Probabilities}

The marginal state probabilities $\left\{P_{i}(k), k=0,1, \ldots, N_{1}\right\}, i=1,2, \ldots, R$, can be determined as follows:

Let us define

$$
\begin{aligned}
& S_{j}^{i N_{1}}=\left\{S / S \in Q, 0 \leq n_{j} \leq N_{j}, j=1, \ldots, R, \& \sum_{i \neq j=1}^{R} n_{j} \leq N_{1}\right\}, \\
& A_{j}^{i v}=\left\{S / S \in Q, 0 \leq n_{j} \leq v ; n_{j} \leq N_{j}, j=1, \ldots, R, \& \sum_{i \neq j=1}^{R} n_{j}=v\right\}
\end{aligned}
$$

$\forall j, i=1,2, \ldots, R, i \geq j \& v=0,1, \ldots, N_{1}$. Using the definition of $P\left(S_{0}\right)$ and $P(S)$, it follows that

$$
\begin{aligned}
& P_{i}(0)=P\left(S_{0}\right)+\sum_{i \neq j=1}^{R}\left(\sum_{\substack{S \in Q_{j} \\
n_{i}=0}} P(S)\right)=P\left(S_{0}\right)+\sum_{i \neq j=1}^{R}\left(\sum_{\substack{S \in S_{j}^{i N_{1}} \\
n_{j} \geq 1}} P(S)\right), \\
& =\frac{1}{Z}\left(1+\sum_{i \neq j=1}^{R} g_{j} \xi_{j} x_{j} \sum_{v=0}^{N_{j}-1} y_{j}^{\delta(v)}\left(\sum_{S \in A_{j}^{i v}} x_{j}^{n_{j}} \prod_{\substack{r \neq i=1 \\
r \neq j}}^{R} x_{r}^{n_{r}} \xi_{r}^{h_{r}(S)}\right)\right), \\
& P_{i}(k)=\sum_{i \neq j=1}^{R}\left(\sum_{\substack{S \in Q_{j} \\
n_{i}=k}} P(S)\right)+\sum_{\substack{S \in Q_{i} \\
n_{i}=k}} P(S) \\
& =\sum_{i \neq j=1}^{R}\left(\sum_{\substack{S \in S_{j}^{i N_{1}} \\
n_{j} \geq 1 \\
n_{i}=k}} P(S)\right)+\sum_{\substack{S \in S_{i}^{i N_{1}} \\
n_{i}=k}} P(S) \\
& =\frac{1}{Z} \xi_{i} x_{i}^{k}\left(\sum_{i \neq j=1}^{R} g_{j} \xi_{j} x_{j} \sum_{v=0}^{\min \left(N_{j}-1, N_{1}-k-1\right)} y_{j}^{\delta(v)} \sum_{S \in A_{j}^{i v}} x_{j}^{n_{j}} \prod_{\substack{r \neq i=1 \\
r \neq j}}^{R} x_{r}^{n_{r}} \xi_{r}^{h_{r}(S)}\right. \\
& \left.+g_{i} \sum_{v=0}^{N_{1}-k} y_{j}^{\delta(v)} \sum_{S \in A_{i}^{i v} v} \prod_{r \neq i=1}^{R} x_{r}^{n_{r}} \xi_{r}^{h_{r}(S)}\right), 1 \leq k \leq N_{1} .
\end{aligned}
$$

For a cost-effective computation of marginal probabilities, recursive formulae can be derived for the complex sums of expressions (25) and (26) as follows: 
Removing the $i$ th class in eq. (18), $C_{i}^{(j)}(v)$ is expressed by

$$
C_{i}^{(j)}(v)= \begin{cases}\sum_{S \in A_{j}^{i v}} x_{j}^{n_{j}} \prod_{\substack{r \neq i=1 \\ r \neq j}}^{R} x_{r}^{n_{r}} \xi_{r}^{h_{r}(S)}, & j \neq i \\ \sum_{S \in A_{i}^{i v} v} \prod_{r \neq i=1}^{R} x_{r}^{n_{r}} \xi_{r}^{h_{r}(S)}, & j=i\end{cases}
$$

where

$$
G_{i}^{(j)}(z)=\sum_{v=0}^{\infty} C_{i}^{(j)}(v) z^{v}
$$

The coefficients $C_{i}^{(j)}(v)$ can be determined via the following recursive formulae:

$$
C_{i}^{(j)}(v)= \begin{cases}C^{(j)}(v)-x_{i} C^{(j)}(v-1)+\left(1-\xi_{i}\right) x_{i} C_{i}^{(j)}(v-1) & \\ +\xi_{i} x_{i}^{N_{i}+1} C_{i}^{(j)}\left(v-N_{i}-1\right), & i \neq j \\ C^{(j)}(v)-x_{i} C^{(j)}(v-1)+x_{i}^{N_{i}} C^{(j)}\left(v-N_{i}\right), & i=j\end{cases}
$$

with initial conditions $C_{i}^{(j)}(v)= \begin{cases}0, & v<0 \\ 1, & v=0\end{cases}$

By substituting these recursive formulae (29) in (25) and (26), the following expressions for marginal probabilities are obtained:

$$
\begin{aligned}
P_{i}(0)= & \frac{1}{Z}\left(1+\sum_{i \neq j=1}^{R} g_{j} \xi_{j} x_{j}\left(\sum_{v=0}^{N_{j}-1} y_{j}^{\delta(v)} C_{i}^{(j)}(v)\right)\right), \\
P_{i}(k)= & \frac{1}{Z} x_{i}^{k} \xi_{i}\left(\sum_{i \neq j=1}^{R} g_{j} \xi_{j} x_{j}\left(\sum_{v=0}^{\min \left(N_{j}-1, N_{1}-k-1\right)} y_{j}^{\delta(v)} C_{i}^{(j)}(v)\right)\right. \\
& \left.+g_{i} \sum_{i \neq j=1}^{R}\left(\sum_{v=0}^{N_{1}-k} y_{j}^{\delta(v)} C_{i}^{(i)}(v)\right)\right), k=1, \ldots, N_{i} .
\end{aligned}
$$

\subsection{Marginal Mean Queue Lengths}

The marginal mean queue lengths (MQLs) $\left\{L_{i}, 1,2, \ldots, R\right\}$ and associated Lagrangian coefficients $\left\{x_{i}\right\}$ satisfy the relationship $L_{i}=\left(x_{i} / Z\right) \partial Z / \partial x_{i}, i=$ $1,2, \ldots, R$ (Jaynes 1957a). Defining appropriate $\mathrm{z}$-transform and after some manipulations, it follows that

$$
L_{i}=\frac{x_{i}}{Z}\left(x_{i} g_{i} \xi_{i} \sum_{v=0}^{N_{1}-1} y_{i}^{\delta(v)} C_{x_{i}}^{(i)}(v)\right), i=1,2, \ldots, R
$$


where $\delta(v)=1$, if $v=N_{1}-1$ and 0 otherwise, and

$$
C_{x_{i}}^{(i)}(v)=x_{i} C_{x_{i}}^{(i)}(v-1)+\frac{1}{x_{i}} C^{(i)}(v), v=1,2, \ldots, N_{1}-1,
$$

with initial condition: $C_{x_{i}}^{(i)}(0)=C^{(i)}(1) / x_{i}$. $C^{(i)}(v)$ values which can be calculated using (19).

\section{OPEN QUEUEING NETWORKS}

Consider an arbitrary open queueing network with $M$ single server queueing stations, $R$ distinct classes of cells, HOL scheduling discipline, PBS scheme with thresholds $\left\{N_{k i}, i=1, \ldots, R, k=1, \ldots, M\right\}$ and RS-RD blocking mechanism. Let

$a_{k i m j}$ be the transition probability (first order Markov chain) that a class $i$ cell transmitted from station $k$ attempts to join station $m$ as class $j$,

$\hat{a}_{k i m j}$ be the effective transition probability,

$a_{k i 0}$ be the transition probability that a cell of class $i$ leaves the network upon finishing transmission at station $k$,

$\Lambda_{0 k i}, C_{a 0 k i}^{2}$ be the mean rate and SCV of the external interarrival process of class $i$ cells into station $k$, respectively,

$\Lambda_{k i}, C_{a k i}^{2}$ be the mean rate and SCV of the overall actual interarrival process of class $i$ cells at station $k$, respectively,

$\lambda_{k i}, \hat{C}_{a k i}^{2}$ be the mean rate and SCV of the overall effective interarrival process of class $i$ cells at station $k$, respectively,

$\mu_{k i}, C_{s k i}^{2}$ be the mean rate and SCV of the actual service process of class $i$ at station $k$, respectively,

$\hat{\mu}_{k i}, \hat{C}_{s k i}^{2}$ be the mean rate and SCV of the effective service process of class $i$ cells at station $k$, respectively,

$\pi_{k i m j}$ be the blocking probability that a cell of class $i$ upon its service completion (call it "completer") from station $k$ will be blocked by station $m$, as class $j$,

$\pi_{k i}$ be the blocking probability that a completer from any station $m, m \neq k$ of class $i$ is blocked by station $k$

$\pi_{0 k i}$ be the blocking probability that an external arrival of class $i$ is blocked by station $k$

$\pi_{c k i}$ be the blocking probability that a completer cell of class $i$ will be blocked by a downstream station.

\subsection{The ME Product-Form Solution}

Let at any given time $n_{i k}$ be the number of cells of class $i$ at queue $k, \mathbf{S}_{k}=$ $\left(n_{k 1}, n_{k 2}, \ldots, n_{k R}, c\right)$ be the state of queue $k$, where $c$ represents the class of 
a cell in service, $\mathbf{S}=\left(\mathbf{S}_{1}, \mathbf{S}_{2}, \ldots, \mathbf{S}_{M}\right)$ be the state of the network, and $\mathbf{Q}_{\mathbf{k}}, \mathbf{Q}$ be the sets of all feasible states $\mathbf{S}_{\mathbf{k}}$ and $\mathbf{S}$, respectively.

The form of the ME solution $P(\mathbf{S}), \mathbf{S} \in \mathbf{Q}$, subject to normalisation and marginal constraints of the type (2) - (6), can be clearly established in terms of the product-form approximation

$$
P(\mathbf{S})=\frac{1}{Z} \prod_{k=1}^{M}\left(\prod_{i=1}^{R} x_{i}^{n_{i}(\mathbf{S})} \xi_{i}^{h_{i}(\mathbf{S})} \sum_{s=1 \wedge n_{s}>0}^{R} g_{s} y_{s}^{f_{s}(\mathbf{S})}\right),
$$

where $Z$ is the normalising constant and $\left\{g_{k i}, \xi_{k i}, x_{k i}, y_{k i}\right\}$ are the Lagrangian coefficients that corresponds to constraints (3) - (6), respectively. Defining $Z_{k}, k=1,2, \ldots, M$, as

$$
Z_{k}=\sum_{\mathbf{S}_{\mathbf{k}} \in \mathbf{Q}_{\mathbf{k}}}\left(\prod_{i=1}^{R} x_{i}^{n_{i}(\mathbf{S})} \xi_{i}^{h_{i}(\mathbf{S})} \sum_{s=1 \wedge n_{s}>0}^{R} g_{s} y_{s}^{f_{s}(\mathbf{S})}\right),
$$

expression (34) can be written as the product of the marginal probabilities $P_{k}\left(\mathbf{S}_{\mathbf{k}}\right), \mathbf{S}_{\mathbf{k}} \in \mathbf{Q}_{\mathbf{k}}$, i.e.,

$$
P(\mathbf{S})=\prod_{k=1}^{M} P_{k}\left(\mathbf{S}_{\mathbf{k}}\right)
$$

where $P\left(\mathbf{S}_{\mathbf{k}}\right)$ is the marginal ME solution of queue $k$,

$$
P\left(\mathbf{S}_{\mathbf{k}}\right)=\frac{1}{Z_{k}} \prod_{i=1}^{R} x_{i}^{n_{i}\left(\mathbf{S}_{\mathbf{k}}\right)} \xi_{i}^{h_{i}\left(\mathbf{S}_{\mathbf{k}}\right)} \sum_{s=1 \wedge n_{s}>0}^{R} g_{s} y_{s}^{f_{s}\left(\mathbf{S}_{\mathbf{k}}\right)},
$$

(c.f., (8)).

\subsection{Queue-By-Queue Decomposition Algorithm}

The ME queue-by-queue decomposition algorithm for the approximate analysis of arbitrary open QNMs with single server queueing stations, $R(R>1)$ HOL priority classes and PBS scheme under RS-RD blocking is described below. It is an extension of the earlier ME algorithm of (Kouvatsos and Denazis 1993) dealing with multiple class open FCFS QNMs with arbitrary topology and repetitive service (RS) blocking. The algorithm incorporates a feedback correction of the transition probabilities in order to mitigate the strong underlying assumption that arrival streams per class are renewal processes. Furthermore, the algorithm describes the computational process of solving the non-linear equations for cell loss $\left\{\pi_{0 k i}\right\}$ and blocking $\left\{\pi_{k i m j}\right\}$ probabilities under appropriate flow formulae for the first two moments of merging, spliting and departing streams (assumed to be known). Note that the probabilities $\left\{\pi_{0 k i}, \pi_{k i m j}\right\}$ generally depend on the effective cell flow bal- 
ance equations for $\left\{\lambda_{0 k i}, \lambda_{k i}\right\}$ and SCV $\hat{C}_{a k i}^{2}$, effective service time parameters $\left\{\hat{\mu}_{k i}, \hat{C}_{s k i}^{2}\right\}$ and overall interarrival time parameters $\left\{\Lambda_{k i}, C_{a k i}^{2}\right\}$.

INPUT DATA

- $M, R$,

- $\forall k, m, k=1,2, \ldots, M, m=0,1, \ldots, M \& \forall i, j=1,2, \ldots, R$,

- $\left\{N_{k}, \Lambda_{0 k i}, C_{a 0 k i}^{2}, \mu_{k i}, C_{s k i}^{2}, a_{k i m j}\right\}$,

Step 1 Feedback correction

For each queue $k, k=1,2, \ldots, M$, and class $i, i=1, \ldots, R$, with $a_{k i m j}>0$, substitute

$$
\begin{aligned}
& \mu_{k i} \leftarrow \mu_{k i}\left(1-a_{k i m j}\right) \\
& C_{s k i}^{2} \leftarrow a_{k i k i}+\left(1-a_{k i k i}\right) C_{s k i}^{2} \\
& a_{k i m j} \leftarrow \begin{cases}0 & , k=m \\
a_{k i m j} /\left(1-a_{k i k i}\right) & , m \neq k, \forall m=1,2, \ldots, M\end{cases}
\end{aligned}
$$

Step 2 Initialize $\pi_{0 k i}, \& \pi_{k i m j} \leftarrow$ any value in $(0,1), \forall k, m=1,2, \ldots, M$ and $\forall i, j=1,2, \ldots, R$,

Step 3 Solve the system of non-linear equations $\left\{\pi_{0 k i}, \pi_{k i m j}\right\}$,

Step 3.1 Calculate effective flow transition probabilities $\left\{\hat{a}_{k i m j}\right\}$ :

$$
\begin{aligned}
& \hat{a}_{k i m j}=a_{k i m j}\left(1-\pi_{k i m j}\right) /\left(1-\pi_{c k i}\right) \\
& \hat{a}_{k i 0}=a_{k i 0} /\left(1-\pi_{c k i}\right) \\
& \pi_{c k i}=\sum_{k \neq m=1}^{M} \sum_{j=1}^{R} a_{k i m j} \pi_{k i m j}, \forall k, m, i
\end{aligned}
$$

Step 3.2 Calculate effective cell flow balance equations:

$$
\begin{aligned}
& \lambda_{0 k i}=\Lambda_{0 k i}\left(1-\pi_{0 k i}\right) \\
& \lambda_{k i}=\lambda_{0 k i}+\sum_{m=1}^{M} \sum_{j=1}^{R} \hat{a}_{m j k i} \lambda_{m j}, \forall k, m, i
\end{aligned}
$$

Step 3.3 Calculate the effective service parameters, $\left\{\hat{\mu}_{k i}, \hat{C}_{s k i}^{2}\right\}$

$$
\begin{aligned}
& \hat{\mu}_{k i}=\mu_{k i}\left(1-\pi_{c k i}\right), \forall k, m, i \\
& \hat{C}_{s k i}^{2}=\pi_{c k i}+\left(1-\pi_{c k i}\right) C_{s k i}^{2}
\end{aligned}
$$

Step 3.4 Calculate overall interarrival parameters, $\left\{\Lambda_{k i}, C_{a k i}^{2}\right\}$ 


$$
\begin{aligned}
& C_{a k i}^{2}=\frac{\hat{C}_{a k i}^{2}-\pi_{k i}}{1-\pi_{k i}} ; \\
& \Lambda_{k i}=\lambda_{k i} /\left(1-\pi_{k i}\right), \forall k, i \\
& \hat{C}_{a k i}^{2}=G_{k i}\left(\lambda_{m i}, C_{a m i}^{2}, \mu_{m i}, C_{s m i}^{2}, a_{k i m j}\right) \\
& \pi_{k i}=\frac{\Lambda_{0 k i} \pi_{0 k i}+\sum_{m=1}^{M} \sum_{j=1}^{R}\left(\hat{a}_{m j k i} \lambda_{m j k i} \pi_{m j k i} /\left(1-\pi_{m j k i}\right)\right)}{\Lambda_{0 k i}+\sum_{m=1}^{M} \sum_{j=1}^{R}\left(\hat{a}_{m j k i} \lambda_{m j} /\left(1-\pi_{m j k i}\right)\right)}
\end{aligned}
$$

where $G_{k i}$ is a suitable flow superposition function.

Step 3.5 Obtain new values for $\left\{\pi_{0 k i}, \pi_{m j k i}\right\}$, by applying Newton Raphson method,

Step 4 Calculate $C_{d k i}^{2}$, the SCV of the interdeparture times

$$
C_{d k i}^{2}=F_{k i}\left(\lambda_{k i}, C_{a k i}^{2}, \mu_{k i}, C_{s k i}^{2}\right)
$$

where $F_{k i}$ is a suitable flow interdeparture function.

Step 5 Calculate new value for $C_{a k i}^{2}$

Step 6 Return to Step 3 until convergence of $C_{d k i}^{2}$.

Step 7 Obtain performance metrics of interest.

The main computational cost of the proposed algorithm is of $O\left\{k R^{3} M^{3}\right\}$, where $\mathrm{k}$ is the number of iterations in step 3 and $\left(\mathrm{R}^{3} \mathrm{M}^{3}\right)$ is the number of operations for inverting the associated Jacobian matrix of the system of nonlinear eq.s $\left\{\pi_{0 k i}, \pi_{k i m j}\right\}$. However, if a quasi-Newton numerical method is employed, this cost can be reduced to be of $O\left\{\mathrm{kR}^{2} \mathrm{M}^{2}\right\}$. Moreover, the existence and unicity of the solution of the non-linear system of Step 3 cannot be shown analytically due to the complexity of the expressions of the cell loss (or blocking) probabilities $\left\{\pi_{0 k i}, \pi_{k i m j}\right\}$; nevertheless, numerical instabilities were never observed during extensive experimentations under any feasible set of initial values. Note that the case of RS-FD blocking can be easily incorporated within the algorithm.

\section{CASE STUDY: THE GE/GE/1/ $\mathrm{N}_{1}, \ldots, \mathrm{N}_{R}$ QUEUE}

In this section, the ME methodology is applied towards the approximate analysis of GE-type open QNMs with $R(R>1)$ HOL priority classes and a PBS scheme under RS-RD blocking mechanism. The $\mathrm{GE} / \mathrm{GE} / 1 / \mathrm{N}_{1}, \ldots, \mathrm{N}_{R}$ queue with GE-type interarrival and service time distributions, in conjunction with GE-type probabilistic arguments, are used to determine the cell loss probabilities $\left\{\pi_{i}, i=1,2, \ldots, R\right\}$ and the Lagrangian coefficients $y_{i}, i=1,2, \ldots, R$ of the ME solution (9). Furthermore, the GE/GE/ $1 / \mathrm{N}_{1}, \ldots, \mathrm{N}_{R}$ queue can play the role of a cost-effective building block, together with GE-type flow formulae for departing and merging streams, towards the approximate analysis of corresponding arbitrary GE-type open QNMs under RS-RD blocking. 


\subsection{Cell Loss Probabilities}

Consider a GE/GE/1/ $\mathrm{N}_{1}, \ldots, \mathrm{N}_{R}$ queue with non-zero interarrival time and service time stage selection probabilities $\sigma_{i}=\left(C_{a i}^{2}+1\right) / 2$ and $r_{i}=\left(C_{s i}^{2}+1\right) / 2$, respectively. Since each arriving stream from class $i$ is a bulk stream at Poisson arriving instants, it is assumed that each of them will see in the queue the same aggregate number of cells as a random observer (n.b., this assumption will strictly be true if the SCVs of the arriving streams are equal). Let that a tagged cell belongs to class $i$ and its bulk finds the system in state $A_{i}^{v}, v=$ $1,2, \ldots, N_{i}$. Thus the number of spaces available to the arriving bulk is $N_{i}-v$, therefore, it follows that

$P$ (a tagged cell is blocked \& the bulk finds the system

$$
\text { in state } \left.A_{j}^{v}, v=1,2, \ldots, N_{i}, j=1,2, \ldots, R\right)=\left(1-\sigma_{i}\right)^{N_{i}-v} P\left(A_{j}^{v}\right) \text {. }
$$

Unconditioning on all those states $A_{j}^{v}$ with $j=1, \ldots, R$, yields

$P($ a class $i$ tagged cell is blocked \& the bulk finds the system

$$
\begin{gathered}
\text { in state, } \left.A_{j}^{v}, j=1,2, \ldots, R\right) \\
=\sum_{j=1}^{R}\left(\sum_{v=1}^{N_{i}-1}\left(1-\sigma_{i}\right)^{N_{i}-v} P\left(A_{j}^{v}\right)\right)+\sum_{j=1}^{R} \sum_{v=N_{i}}^{N_{1}} P\left(A_{j}^{v}\right) .
\end{gathered}
$$

Finally, taking into account the idle state $S_{o}$,

$P$ (a tagged cell is blocked \& the bulk finds the system

$$
\text { in state } \left.S_{o},\right)=\delta_{i}\left(1-\sigma_{i}\right)^{N_{i}} P\left(S_{o}\right) \text {, }
$$

where $\delta_{i}=r_{i} /\left(r_{i}\left(1-\sigma_{i}\right)+\sigma_{i}\right)$. Combining eq.s (38)- (40), the following expression for probability $\pi_{i}$, is obtained.

$$
\pi_{i}=\delta_{i}\left(1-\sigma_{i}\right)^{N} P\left(S_{o}\right)+\sum_{j=1}^{R}\left(\sum_{v=1}^{N_{i}-1}\left(1-\sigma_{i}\right)^{N_{i}-v} P\left(A_{j}^{v}\right)\right)+\sum_{j=1}^{R} \sum_{v=N_{i}}^{N_{1}} P\left(A_{j}^{v}\right)
$$

It is trivial to prove that, $\forall j=1,2, \ldots, R$

$$
P\left(A_{j}^{v}\right)=\frac{1}{Z} g_{j} \xi_{j} x_{j} C_{j}(v-1), \forall v=1, \ldots, N_{1}-1 .
$$


Substituting (42) into (41), yields

$$
\begin{aligned}
\pi_{i}= & \frac{1}{Z} \delta_{i}\left(1-\sigma_{i}\right)^{N_{i}}+\frac{1}{Z} \sum_{j=1}^{R} g_{j} \xi_{j} x_{j}\left(\sum_{v=1}^{N_{i}-1}\left(1-\sigma_{i}\right)^{N_{i}-v} C^{(j)}(v-1)\right) \\
& +\sum_{v=N_{i}}^{N_{1}} P(v),
\end{aligned}
$$

which, finally takes the form

$$
\pi_{i}=\sum_{v=0}^{N_{1}} \delta_{i}(v)\left(1-\sigma_{i}\right)^{\left[N_{i}-v\right]^{+}} P(v),
$$

where $\delta_{i}(v)=\delta_{i}$, if $v=0$ and $\delta_{i}(v)=1, \forall v \geq 1$.

\subsection{Estimation of the Lagrangian Coefficients $\left\{y_{i}\right\}$}

From the flow balance conditions (7) and by using expressions (22), (24)(44), the Lagrangian coefficients, $y_{i}, i=1,2, \ldots, R$, can be determined, after some manipulation, by

$$
\begin{aligned}
& y_{i}= \frac{\rho_{i}}{g_{i} \xi_{i} x_{i} \sum_{v=N_{i}-1}^{N_{1}-1} C^{(i)}(v)}\left(\sum_{j=1}^{R} g_{j} \xi_{j} x_{j}\left(\sum_{v=0}^{N_{1}-2} y_{j}^{\delta(v)} C^{(j)}(v)\right)\right. \\
&+1-\delta_{i}\left(1-\sigma_{i}\right)^{N_{i}}-\frac{1}{\rho_{i}} g_{i} \xi_{i} x_{i} \sum_{v=0}^{N_{i}-2} C^{(i)}(v) \\
&\left.-\sum_{j=1}^{R} g_{j} \xi_{j} x_{j} \sum_{v=1}^{N_{i}-1}\left(1-\sigma_{i}\right)^{N_{i}-v} C^{(j)}(v-1)\right), \\
& i=1,2, \ldots, R .
\end{aligned}
$$

\subsection{Flow Formulae}

The ME approximation suggests a decomposition of the network into individual multiple class $\mathrm{GE} / \mathrm{GE} / 1 / \mathrm{N}_{1}, \ldots, \mathrm{N}_{R}$ queues with merged arrival process and revised service time for each class of cells. In order to implement the $\mathrm{ME}$ solution under an abstract service discipline, the flow processes in the general 
network should be determined. For each queue $k, k=1,2, \ldots, M$, it is assumed that the arriving and departing streams per class $i$ cells, $i=1,2, \ldots, R$, form the renewal processes conforming with GE-type underlying interarrival time and service time distributions. The mean rates and SCVs of the overall interarrival and interdeparture processes of class $i$ at queue $k$ can be determined as follows c.f., (Kouvatsos and Tabet-Aouel 1990):

The Interdeparture Process:

$\lambda_{d k i}=\lambda_{k i}$,

$C_{d k i}^{2}=2\left\langle n_{k i}\right\rangle\left(1-\theta_{k i}\right)+C_{a k i}^{2}\left(1-2 \theta_{k i}\right)$.

The Split Process:

$$
\begin{aligned}
\lambda_{m j k i} & =\lambda_{m j} \hat{a}_{m j k i}, \\
C_{d m j k i}^{2} & =1+\hat{a}_{m j k i}\left(C_{d k i}^{2}-1\right) .
\end{aligned}
$$

The Merging Process:

$$
\begin{aligned}
& \lambda_{k i}=\sum_{m=1}^{M} \sum_{j=1}^{R} \lambda_{m j k i}, \\
& C_{a k i}^{2}=-1+\left(\sum_{m=1}^{M} \sum_{j=1}^{R} \frac{\lambda_{m j k i}}{\lambda_{k i}}\left(C_{d m j k i}^{2}\right)^{-1} \frac{\lambda_{0 k i}}{\lambda_{k i}}\left(C_{a 0 k i}^{2}+1\right)^{-1}\right)^{-1},
\end{aligned}
$$

for $i, j=1,2, \ldots, R$, and $k, m=1,2, \ldots, M$.

\section{$5 \quad$ NUMERICAL RESULTS}

The credibility of the proposed ME algorithm is illustrated against simulation via two typical numerical tests involving simple multistage interconnection networks with GE-type external interarrival and transmission times and single input/output ports and output queueing. Tables 1-2 present the input data relating to central server and feedforward types of open QNMs, respectively, with three nodes (switching components) and two HOL priority classes of cells under PBS scheme. Cells entering and flowing in the network follow a CPP with geometric batches, whilst for tractability purposes, the GE distribution with SCV equal to 0.5 is also used to model an approximate sense constant service times. Furthermore, two moment flow approximation formulae are used based on the superposition and splitting of GE-type streams as well as the interdeparture time distribution of a stable GE/GE/1 queue at equilibrium (Kouvatsos 1994). For validaton purposes, the following tolerances 
(TOL) have been used:

For throughput, $X$, the TOL is defined by

$$
\left|\frac{\operatorname{SIM}(X)-\operatorname{MEM}(X)}{\operatorname{SIM}(X)}\right|
$$

For the marginal mean queue lengths, $\langle n\rangle$, the TOL is defined by

$$
\left|\frac{\operatorname{SIM}\left(\left\langle n_{k i}\right\rangle\right)-\operatorname{MEM}\left(\left\langle n_{k i}\right\rangle\right)}{\sum_{k=1}^{M} \operatorname{SIM}\left(\left\langle n_{k, i}\right\rangle\right)}\right|
$$

and TOL for queue length distribution, $P(n)$, utilisation, $U$ and cell loss probabilities, $P c$, is defined as follows:

$$
\left|\operatorname{SIM}\left(e_{k i}\right)-\operatorname{MEM}\left(e_{k i}\right)\right|, \text { respectively }
$$

for each class $i$ and station $k$. The results of the validation study are presented in Figures 1-6 which display the error tolerances between ME algorithm and simulation (based on QNAP-2 at 95\% confidence interval (Veran and Potier 1985)) for the performance measures of throughput $X$, mean queue length $\langle n\rangle$, utilisation $U$, idle and full buffer state probabilities and cell loss (or blocking) probabilities. It can be observed that the ME solutions are very comparable to those obtained by corresponding simulation models. Note, however, the credibility of the ME solution gradually deteriorates by increasing the service time SCVs. This behaviour may be attributed to the fact that the underlying interarrival and interdeparture renewal assumptions are further violated. 


\section{Experiment 1}

\section{Table 1}

\begin{tabular}{ll}
\hline Raw data: & $M=3, R=2, N_{1}=10, N_{2}=6$ \\
Queue $1:$ & $\mu_{1}=6.25, \mu_{2}=25, C_{s 1}^{2}=2, C_{s 2}^{2}=3$ \\
& $\lambda_{1}=1, \lambda_{2}=1, C_{a 1}^{2}=3, C_{a 2}^{2}=5$ \\
& $a_{1121}=0.5, a_{1131}=0.5, a_{110}=0, a_{1222}=0.5, a_{1232}=0.5$ \\
& $a_{120}=0$ \\
Queue 2: & $\mu_{1}=3.75, \mu_{2}=5, C_{s 1}^{2}=3, C_{s 2}^{2}=5$ \\
& $a_{2111}=0.4, a_{2131}=0, a_{210}=0.6, a_{2212}=0.4, a_{2232}=0$ \\
& $a_{220}=0.6$ \\
Queue 3: & $\mu_{1}=4, \mu_{2}=12, C_{s 1}^{2}=3, C_{s 2}^{2}=6$ \\
& $a_{3111}=0.3, a_{3121}=0, a_{310}=0.7, a_{3212}=0.3, a_{3222}=0$ \\
& $a_{320}=0.7$
\end{tabular}

\section{Experiment 2}

\section{Table 2}

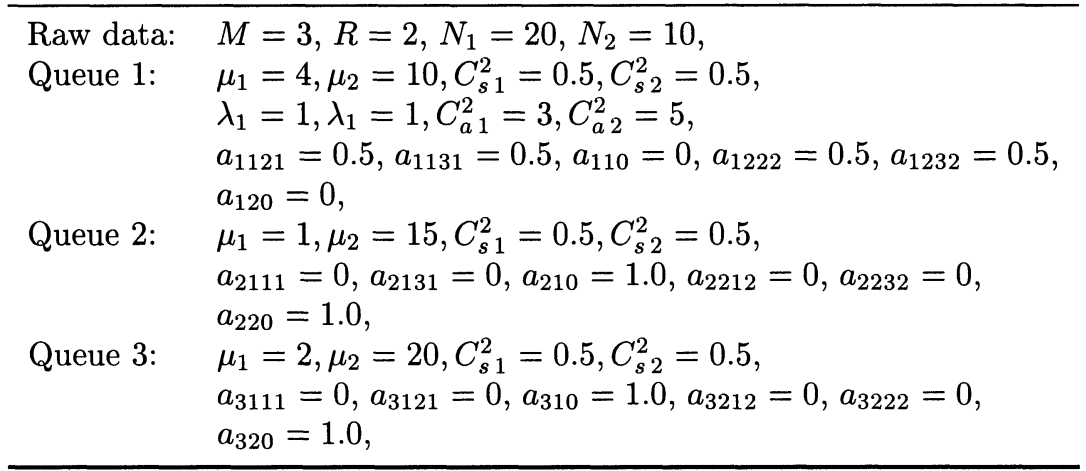

\section{CONCLUSIONS}

A new ME product-form approximation is proposed for arbitrary open QNMs with HOL priority classes under PBS scheme and RS-RD blocking. This solution is implemented in terms of an iterative queue-by-queue decomposition algorithm for the cost-effective estimation of typical performance metrics such as cell loss and state probabilities, throughputs, utilisations and mean queue lengths. The $\mathrm{G} / \mathrm{G} / 1 / \mathrm{N}_{1}, \ldots, \mathrm{N}_{R}$ queue is analysed recursively via entropy maximisation and the generating function approach and plays the role of an efficient building block in the solution process. Numerical experiments, focusing on simple buffered interconnection networks under GE-type bursty traffic and two moment flow approximation formulae, illustrate the high level of accuracy of the ME algorithm against simulation. The ME methodology can also be used to study discrete-time QNMs such as those based on the 
EXPERIMENT - 1

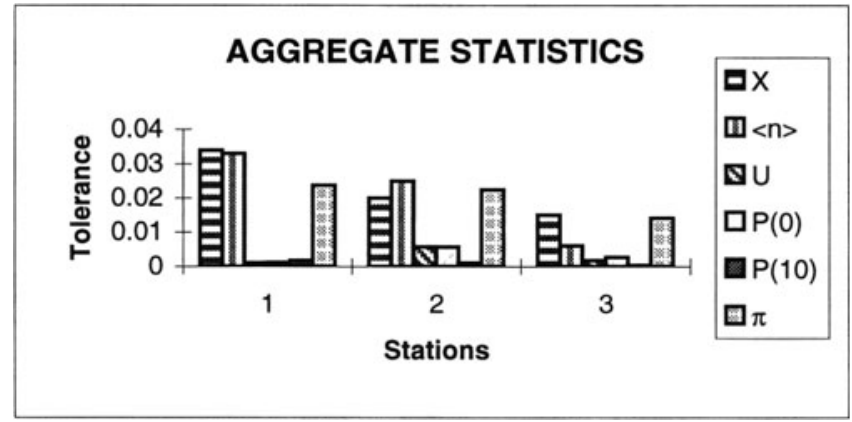

Figure 1: Error tolerances of typical performance measure for all jobs in the network.

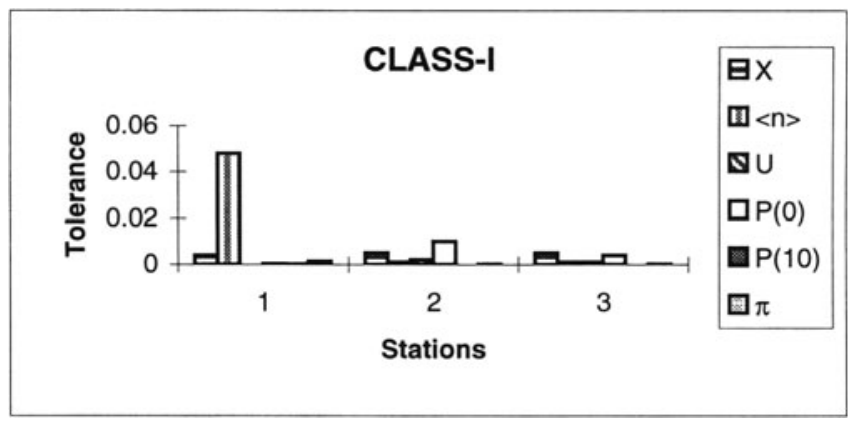

Figure 2: Error tolerances of typical performance measure for Class-I jobs in the network.

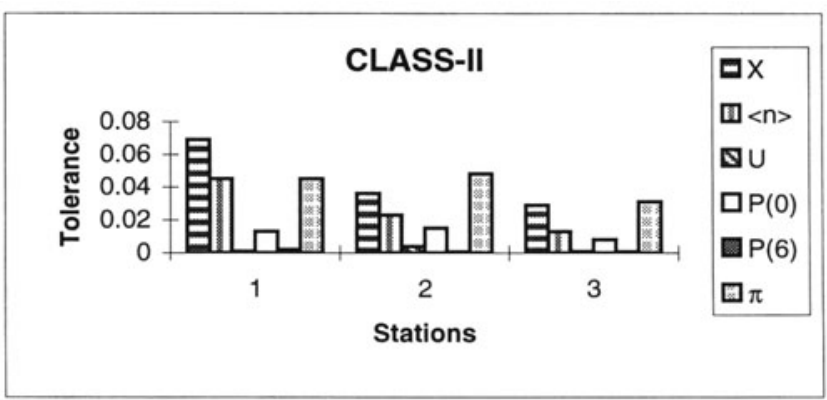

Figure 3: Error tolerances of typical performance measure for Class-II jobs in the network. 
EXPERIMENT - 2

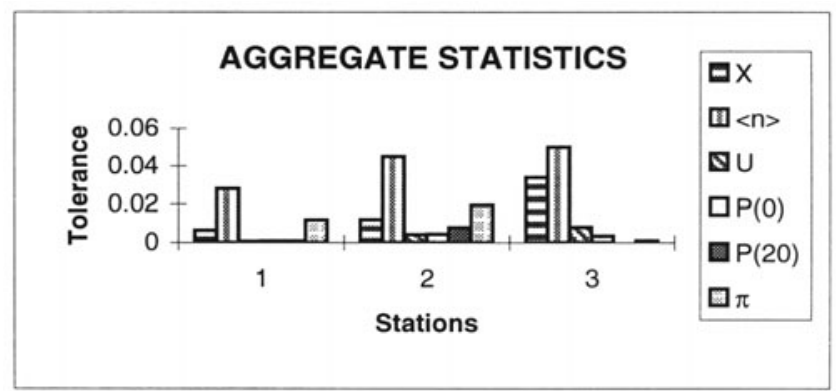

Figure 4: Error tolerances of typical performance measure for all jobs in the network.

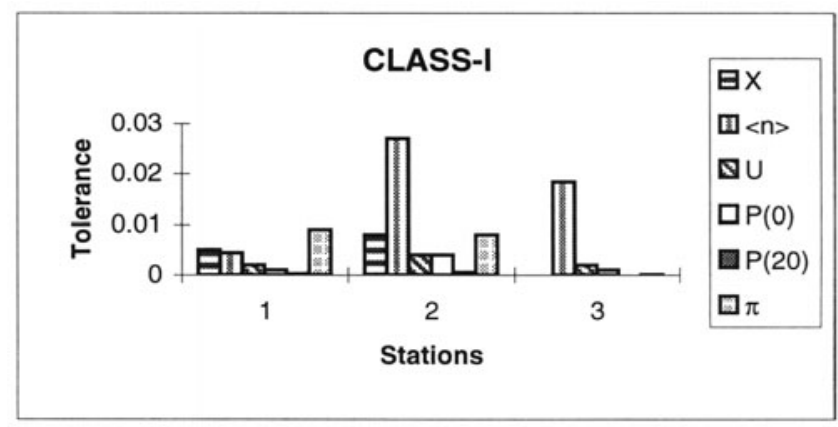

Figure 5: Error tolerances of typical performance measure for Class-I jobs in the network.

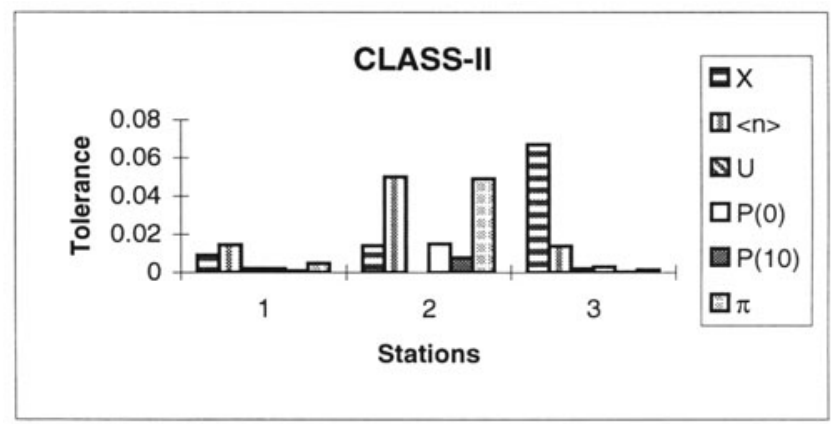

Figure 6: Error tolerances of typical performance measure for Class-II jobs in the network. 
Generalised Geometric (GGeo) distribution (Kouvatsos 1994) and the shifted GGeo (sGGeo) process (Kouvatsos and Fretwell 1995) with applications to multibuffer, shared buffer and shared medium ATM switch architectures with or without correlated traffic under various buffer management policies. Work of this kind is the subject of current studies.

\section{REFERENCES}

Ball, F., Hutchinson, D. and Kouvatsos, D. D. (1996) VBR Video Traffic Smoothing at the AAL SAR Level, Proc. of 4th IFIP Workshop on Performance Modelling and Evaluation of ATM Networks, (ed. D. D. Kouvatsos), Ilkley, 28/1-28/10

Fourneau, J., Pekergin, F. and Taleb, H. (1994) An Application of Stochastic Ordering to the Analysis of the Push-Out Mechanism, Proc. of the Second Workshop on Perf. Modelling and Evaluation of ATM Networks, Bradford, UK, July.

Gravey and G. Hebuterne, G. (1991) Mixing Time and Loss Priorities in a Single Server Queue, Proc. of the International Teletraffic Congress ITC-13, 147-152

Hebuterne and Gravey, A. (1989) A Space Priority Queueing Mechanism for Multiplexing ATM Channels, ITC Specialist Seminar, Adelaide.

Hong, D. and Suda, T. (1991) Congestion Control and Prevention in ATM Networks, IEEE Network Magazine, 10-16

ITU Draft Recommendations 1.150 and 1.361-363 (1990) Broadband Aspect of ISDN-BISDN ATM Functional Characteristics, ATM, Layer Specification for B-ISDN and B-ISDN ATM Adaptation Layer Functional Description/Specification. Geveva, Switzerland.

Jaynes, E.T. (1957a) Information Theory and Statistical Mechanics, Phys. Rev. 106, 620-630

Jaynes, E.T. (1957b) Information Theory and Statistical Mechanics, II Phys. Rev. 108, 171-190

Kouvatsos, D.D. (1986) Maximum Entropy and the G/G/1/N Queue, Acta Informatica, Vol. 23, 545-565

Kouvatsos, D.D. (1994) Entropy Maximisation and Queueing Network Models, Annals of Operation Research Vol. 48, 63-126

Kouvatsos, D.D.and Tabet-Aouel, N. (1990) Product-Form Approximations for an Extended Class of General Closed Queueing Networks, Performance '90, (ed. P. J. B. King, I. Mitrani and R. J. Pooley), Proc. of the 14th IFIP WG 7.3 International Symposium on Computer Performance Modelling, Measurement and Evaluation, Edinburgh.

Kouvatsos, D.D.and Denazis, S.G. (1993) Entropy Maximised Queueing Networks with Blocking and Multiple Job Classes, Performance Evalua- 
tion, Vol. 17, 189-205.

Kouvatsos, D.D.and Fretwell, R.J. (1995) Closed Form Performance Distributions of a Discrete Time GI ${ }^{\mathrm{G}} / \mathrm{D} / 1 / \mathrm{N}$ Queue with Correlated Traffic, Enabling High Speed Networks (ed. S. Fdida and R.O. Onvural), IFIP Publication, Chapman and Hall, ISBN 041273250 5, 141-163

Kroner, H. (1989) Comparative Performance Study of Space Priority Mechanisms for ATM Networks, Proc. IEEE INFOCOM '90, 1136-1143

Li, S. Q. (1989) Overload Control in a Finite Message Storage Buffer, IEEE Trans. Commun., Vol. COMP-37 No. 12, 1330-1338

Lin, A. Y. M. and Silvester, J. A. (1993) Priority Queueing Strategies and Buffer Allocation Protocols for Traffic Control at an ATM Integrated Broadband Switching System, IEEE JSCA, Vol. 9, No. 9.

Nillson, A. A., Lai, F. and Perros, H. G. (1990) A Queueing Model of a Bufferless Synchronous Clos ARM Switch with Head-of-Line Priority and Push-out, Technical Report, Center for Communication and Signal Processing, North Carolina State University, USA.

Sumita, S. and Ozawa, T. (1988) Achievability of Performance Objectives in ATM Sitch Nodes, Proc. of the International Seminar on Performance of Distributed and Parallel Systems, Kyoto, Japan.

Veran, M. and Potier, D. (1985) QNAP-2: A Portable Environment for Queueing Network Modelling Techniques and Tools for Performance Analysis, (ed. D. Potier), North Holland, 25-63

Williams, A. C. and Bhandiwad, R. A. (1976) A Generating Function Approach to Queueing Network Analysis of Multiprogrammed Computers, Networks Vol. 6, 1-22

Yin, N., Li, S. Q. and Stern, T. E. (1990) Congestion Control for Packet Voice by Selective Packet Discarding, IEEE Trans. Commun., Vol. 38, No. $5,674-683$ 